\title{
Treatment of Perichondritis with a Quinolone Derivative-Norfloxacin
}

JULIAN M. THOMAS, M.D.

NEIL A. SWANSON, M.D.

Abstract. Auricular perichondritis is an uncommon complication following surgery of the external ear. Pseudomonas aeruginosa is the most commonly associated pathogen. Presented is a patient with auricular perichondritis successfully treated with norfloxacin, a newly available, orally administered antibiotic with antipseudomonal activity.

\section{INTRODUCTION}

Perichondritis of the external ear represents an uncommon but potentially serious consequence of auricular trauma and/or surgery. The most common bacterial pathogen associated with auricular perichondritis is Pseudomonas aeruginosa. ${ }^{1,2}$ In more severe cases, hospitalization is required for intensive local care (debridement, drains for infusion of local antibiotics), as well as intravenous antibiotics with antipseudomonal activity. Before the introduction of 4-quinolone antibiotics, there had not been an oral antibiotic with good antipseudomonal activity. Norfloxacin, a recently available 4-quinolone, is reported to have excellent antipseudomonal activity after oral administration. ${ }^{3}$ Reported here is a patient who, following Mohs surgery, had auricular perichondritis successfully treated as an outpatient with norfloxacin.

Julian M. Thomas, M.D., is Assistant Professor of Dermatology, UAB/University Station, Department of Dermatology, Birmingham, Alabama.

Neil A. Swanson, M.D., is Associate Professor of Dermatology, The University of Michigan Medical Center, Department of Dermatology, Ann Arbor, Michigan.

Reprints are not available.

J. Dermatol. Surg. Oncol. 14:4 April 1988

\section{CASE REPORT}

A 64-year-old white man presented to the Cutaneous Surgery and Oncology Unit, Department of Dermatology, at the University of Michigan with a history of recurrent basal cell carcinoma involving the concha of the right auricle. The tumor had been initially treated by electrodesiccation and curettage in 1957. In 1959, the tumor was treated by excision and a third recurrence was treated in 1970 by $x$-ray therapy. In 1984, Mohs micrographic surgery was employed to treat the fourth recurrence. Unfortunately the basal cell carcinoma again recurred, leading to Mohs micrographic surgery on March 30, 1987.

The clinical exam revealed an ill-defined nodule overlying the right concha and measuring $1.5 \times$ $1.3 \mathrm{~cm}$. No palpable lymph nodes were detected and there were no other significant physical findings.

Because of the location and recurrent nature of the tumor, Mohs surgery, fresh tissue technique, was employed. A tumor-free plane was found after two stages with a final defect size of $2.1 \times 1.9 \mathrm{~cm}$ (Fig. 1). Perichondrium was intact and we decided to allow the wound to heal by second intention. Careful patient instructions regarding proper wound care were given.

The patient returned in seven days with complaints of ear pain, tenderness, and swelling, but no complaint of fever or chills. On exam, the entire right auricle was noted to be edematous, erythematous, and tender to touch. A yellow discharge was seen in association with the surgical defect.

A culture of the wound was obtained followed by local debridement and cleaning. The patient was instructed to continue local wound care with dilute 
acetic acid soaks t.i.d. followed by Bacitracin ointment and bandage. He was also started on cephalexin $250 \mathrm{mg}$ P.O. q.i.d.

The patient returned in three days with no discernible improvement in his clinical condition (Fig. 2). He was afebrile but the ear remained swollen, erythematous, and tender. The culture from his wound grew Pseudomonas aeruginosa which was resistant to cephalexin (Table 1). The organism was sensitive to ticarcillin and gentamycin, which are both administered intravenously. The organism was found also to be sensitive to a new 4-quinolone antibiotic, norfloxacin, recently released for oral administration.

TABLE 1

Wound Culture Right Ear

Culture Result-Numerous Pseudomonas Aeruginosa

\begin{tabular}{lccl}
\hline Susceptibility Test & MIC & $\mathrm{mcg} / \mathrm{ml}$ & Interpretation \\
\hline Amikacin & $<$ & 4.00 & Sensitive \\
Ampicillin & $>$ & 16.00 & Resistant \\
Cefazolin & $>$ & 32.00 & Resistant \\
Cefamandole & $>$ & 32.00 & Resistant \\
Cefoxitin & $>$ & 32.00 & Resistant \\
Chloramphenicol & $>$ & 16.00 & Resistant \\
Gentamicin & & 2.00 & Sensitive \\
Piperacillin & $<$ & 4.00 & Sensitive \\
Ticarcillin & & 32.00 & Intermediate \\
Trimethoprim/Sulfamethoxazol & $<$ & $4.00 / 76.00$ & Resistant \\
Tobramycin & $<$ & 2.00 & Sensitive \\
Imipenem & $<$ & 4.00 & Sensitive \\
Timentin & & 16.00 & Sensitive \\
Ceftriaxone & $<$ & 16.00 & Sensitive \\
Ceftazidime & $<.00$ & Sensitive \\
Norfloxacin & $<$ & $16.00^{*}$ & Sensitive \\
\hline
\end{tabular}

*Urine

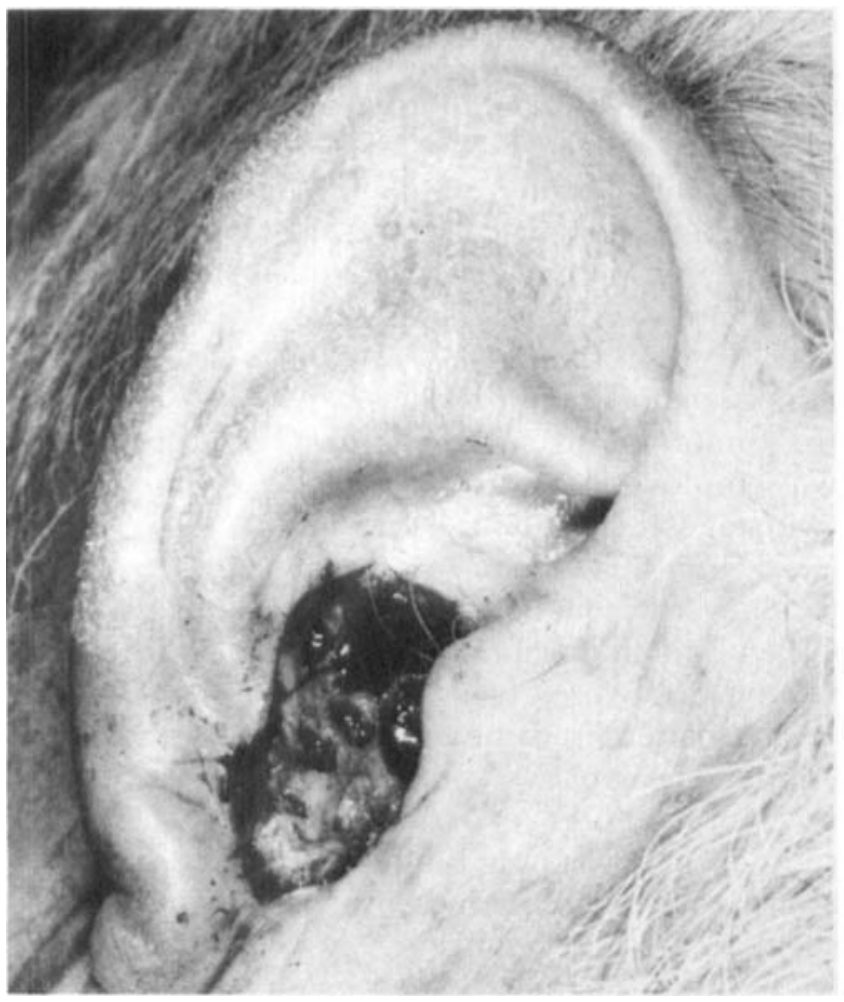

FIGURE 1. Post Mohs defect for recurrent basal cell carcinoma of the right external ear.

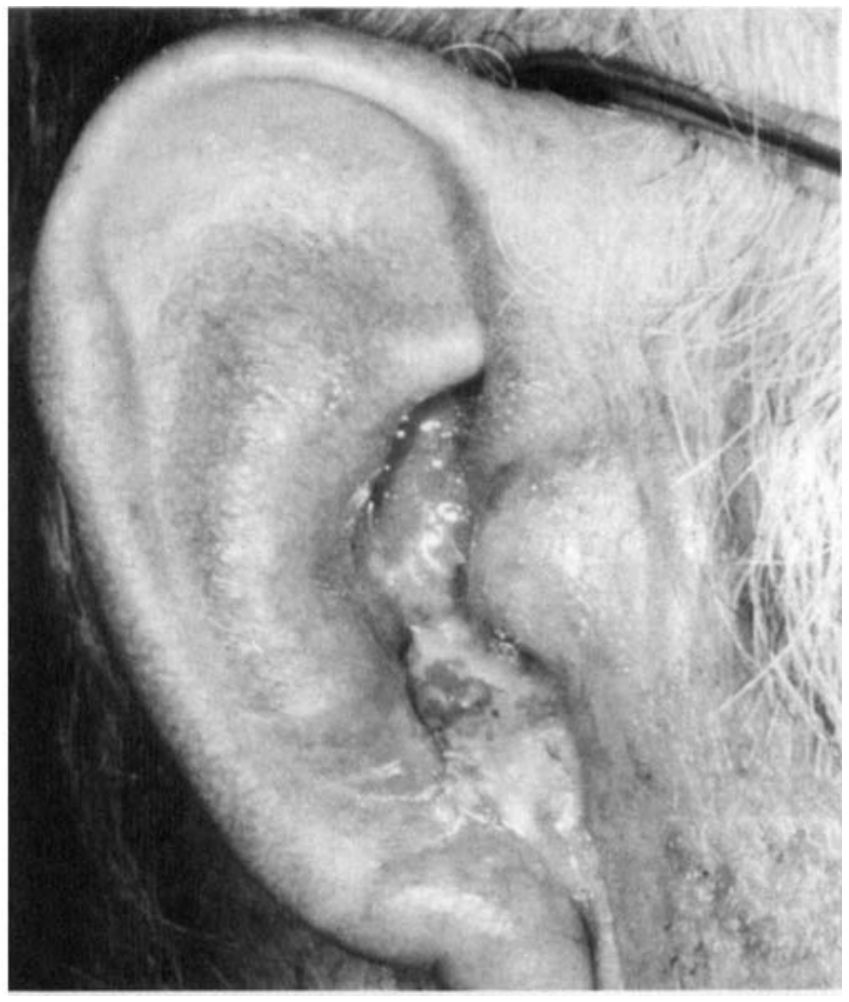

FIGURE 2. Inflammation, edema, and purulent exudate at the site of surgery 7 days postoperatively.

J. Dermatol. Surg. Oncol. 14:4 April 1988 


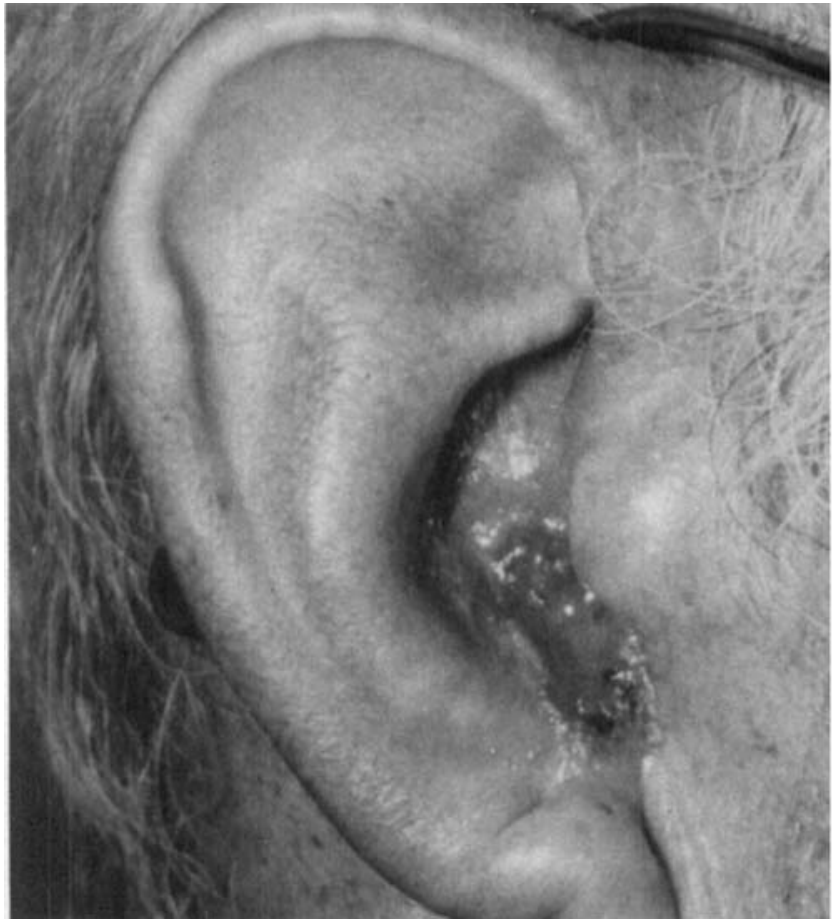

FIGURE 3. Marked improvement after 2 weeks of norfloxacin $400 \mathrm{mg}$ P.O. b.i.d.

Cephalexin was discontinued on day 3 and the patient began norfloxacin $400 \mathrm{mg}$ P.O. b.i.d. The patient experienced marked subjective improvement within 48 hours. On his follow-up exam 7 days after initiation of norfloxacin, the erythema, swelling, and tenderness were negligible (Fig. 3). The norfloxacin was given for a total of 21 days and the patient experienced a subsequently uneventful convalescence (Fig. 4).

\section{DISCUSSION}

While perichondritis of the auricle is an uncommon complication following surgical procedures, it can be difficult to manage. Meticulous wound care and broad spectrum antibiotics have been the mainstays of therapy for less severe cases, whereas advanced cases have required hospitalization for more intensive care, including systemically administered antibiotics. Because of the high frequency of Pseudomonas isolates in auricular perichondritis, it is surprising that more cases have not required intravenous antibiotics. The introduction of the 4-quinolone class of antibiotics marks the first antibiotics available per oral route for use in the treatment of systemic Pseudomonal infections.

Norfloxacin was the first 4-quinolone antibiotic available in the United States. Norfloxacin and

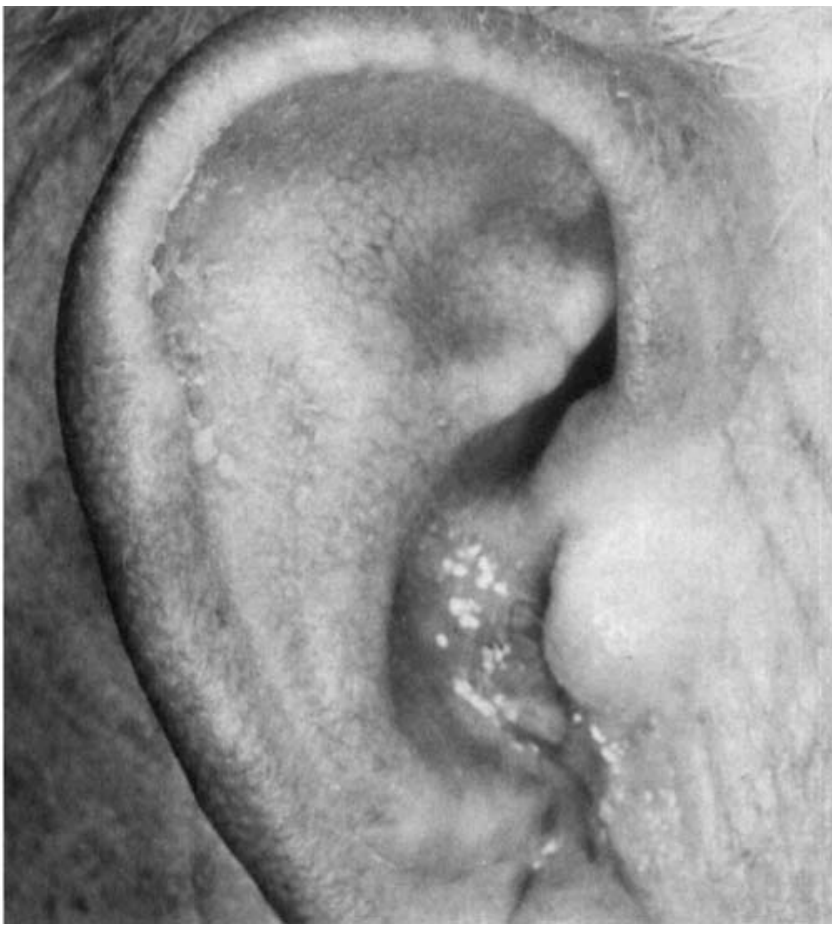

FIGURE 4. Wound 2 weeks following norfloxacin therapy.

other agents in this class were initially evaluated for use in urinary tract infections. It was found that this new class of antibiotics exhibits broad activity against many gram positive and gram negative bacteria, including Pseudomonas species and Staphylococcus aureus. ${ }^{4,5}$ Norfloxacin is available only as an oral preparation and is generally well tolerated. Side effects have been uncommon $(2-4 \%)$, the most common complaints being nausea, headache, and light-headedness.

While norfloxacin to our knowledge has not been reported for the treatment of auricular perichondritis, its spectrum of activity, low incidence of adverse reactions, and oral administration prompted its use in our patient. The excellent response obtained in this patient merits further investigation of this drug in the treatment of auricular perichondritis.

\section{REFERENCES}

1. Martin R, et al. Perichondritis of the ear. Laryngoscope 86:664-673, 1976

2. Goel TK, et al. Management of the acutely burned ear. Burns 9:218-221, 1983.

3. Abramowicz $M$, et al. Norfloxacin (Noroxin). Med Lett 29:25-27, 1987.

4. Cynamon $\mathrm{MH}$, et al. The role of 4-quinolones in the treatment of infections. Comp Ther 13:37-43, 1987.

5. Holmes B, et al. Norfloxacin: A review of its antibacterial activity, pharmacokinetic properties and therapeutic use. Drugs 300:482-513, 1985. 


\section{PSORCON N}

\section{(diflorasone diacetate) $15,30,60$ gsizes}



\section{Dermik}

Dedicated to Dermatology

Dermik Laboratories, Inc.. Blue Bell, PA 19422
Prognosis for Recurrent Stage I Malignant Melanoma. DS Reintgen, $R$ Vollmer, CY Tso, HF Siegler. Arch Surg 122:1338-1342, 1987.

This retrospective study analyzed 1,504 (35.9\%) patients out of 4,185 who had metastases after treatment of their initial malignant melanoma. Initial recurrence site was local (skin, in-transit, or regional lymph nodes) and made up $62.5 \%$ of head and neck primaries, $77.3 \%$ of trunkal tumors, and $85.6 \%$ of extremity melanomas. One third of the head and neck melanomas had their initial recurrence systemically. There was an increased risk of initial recurrence even after a 5-year latency period. Survival figures dropped from 88 to $42 \%$ in this group. Survival by site of recurrence was greatest for local disease $>$ lymph node disease $>$ systemic involvement. Statistical analysis of factors contributing to a worse prognosis after a recurrence were thickness of lesions, ulceration, a short disease-free interval, wide-local excision only, a primary extremity tumor, and older age of the patient.

R. STEVEN PADILLA, M.D.

Refinements in Combined Chemical Peel and Simultaneous Abrasion of the Face. CE Horton, RC Sadove. Ann Plast Surg 19:504-509, 1987.

The authors describe their technique of the use of a Baker's phenol formula chemical peel and immediate dermabrasion of the same facial area via a wire brush, which they have performed in over 500 cases since 1973. They feel the combined approach retains the advantages of both procedures while eliminating some of their disadvantages. Eyelids are treated with peeling alone. One patient developed a hypertrophic scar which resolved with conservative management.

HUBERT T. GREENWAY, JR., M.D

Zoster-like leiomyomata. PA Guerra, M Polimeni, G Santoro, SP Cannavo, Derm Clin 7:52-56, 1987 (Italian).

The authors report a case of a 35-year-old man with zoster-like multiple cutaneous leiomyomata of pilar origin; the patient had many papulonodular elements that were aching spontaneously. He had recurrent and severe neuralgic attacks of pain provoked by physical stimulation, emotion, and change of temperature.

YEHUDI M. FELMAN, M.D 